\title{
Fe I/Fe II ionization equilibrium in cool stars: NLTE versus LTE
}

\author{
Lyudmila Mashonkina ${ }^{1,2}$, Thomas Gehren ${ }^{2}$, Jianrong Shi ${ }^{3}$, \\ Andreas Korn ${ }^{4}$, and Frank Grupp ${ }^{2}$ \\ ${ }^{1}$ Institute of Astronomy, Russian Academy of Science, \\ Pyatnitskaya 48, 119017 Moscow, Russia \\ email: lima@inasan.ru \\ ${ }^{2}$ Universitäts-Sternwarte München, \\ Scheinerstr. 1, 81679 München, Germany \\ email: lyuda, gehren, fug@usm.lmu.de \\ ${ }^{3}$ National Astronomical Observatories, Chinese Academy of Sciences, \\ A20 Datun Road, Chaoyang District, Beijing 100012, PR China \\ email: sjr@bao.ac.cn \\ ${ }^{4}$ Department of Physics and Astronomy, Uppsala University, \\ Box 515, 75120 Uppsala, Sweden \\ email: andreas.korn@fysast.uu.se
}

\begin{abstract}
Non-local thermodynamic equilibrium (NLTE) line formation for neutral and singlyionized iron is considered through a range of stellar parameters characteristic of cool stars. A comprehensive model atom for Fe I and Fe II is presented. Our NLTE calculations support the earlier conclusions that the statistical equilibrium (SE) of Fe I shows an underpopulation of Fe I terms. However, the inclusion of the predicted high-excitation levels of Fe I in our model atom leads to a substantial decrease in the departures from LTE. As a test and first application of the Fe I/II model atom, iron abundances are determined for the Sun and four selected stars with well determined stellar parameters and high-quality observed spectra. Within the error bars, lines of Fe I and Fe II give consistent abundances for the Sun and two metal-poor stars when inelastic collisions with hydrogen atoms are taken into account in the SE calculations. For the close-to-solar metallicity stars Procyon and $\beta$ Vir, the difference (Fe II - Fe I) is about 0.1 dex independent of the line formation model, either NLTE or LTE. We evaluate the influence of departures from LTE on Fe abundance and surface gravity determination for cool stars.
\end{abstract}

Keywords. atomic data, line: formation, stars: atmospheres

\section{Introduction}

Iron plays an outstanding role in studies of cool stars thanks to quite numerous lines in the visible spectrum, which are easy to detect even in very metal-poor stars. Iron serves as a reference element for all astronomical research related to stellar nucleosynthesis and chemical evolution of the Galaxy. Iron lines are used to determine the surface gravity, $\log \mathrm{g}$, and the microturbulence $\xi$ of stellar atmospheres. In the atmosphere with $T_{\text {eff }}>$ $4500 \mathrm{~K}$, neutral iron is a minority species. The ionization equilibrium between Fe I and Fe II and the excitation equilibrium of Fe I easily deviate from thermodynamic equilibrium. Since the beginning of the 1970s a number of studies attacked the problem of non-local thermodynamic equilibrium (NLTE) for Fe (e.g., Athay \& Lites (1972), Thevenin \& Idiart (1999), Gehren et al. (2001)). However, a consensus on the expected magnitude of the NLTE effects was not reached.

In this study, we update the model atom of Fe I-II treated by Gehren et al. (2001) (hereafter Paper I) and apply it to analysis of the Fe spectrum in the Sun and selected 
cool stars with the aim of empirically constraining the role of inelastic collisions with hydrogen atoms in the SE of Fe I-II.

\section{The Fe model atom}

In all previous NLTE calculations, the model atom of Fe I was build using measured energy levels. The experimental analysis of Nave et al. (1994) with later updates provided 965 energy levels for Fe I. A comparison with the calculated Fe I atomic structure (Kurucz (2007)) reveals that the system of measured levels is nearly complete below excitation energy, $E_{\text {exc }}, 5.6 \mathrm{eV}$, however, laboratory experiments do not see most of the highexcitation levels with $E_{\text {exc }}>7.1 \mathrm{eV}$. As already shown in the first NLTE studies, the main NLTE mechanism for Fe I is the overionization of low-excitation levels by ultraviolet radiation. The role of high-excitation levels is to compensate, in part, for population losses via collisional coupling to the large continuum reservoir, with subsequent spontaneous transitions down to low-excitation levels. Therefore, the system of levels in the model atom of $\mathrm{Fe} \mathrm{I}$ has to be fairly complete at least up to $0.5 \mathrm{eV}$ (mean kinetic energy of electrons in the atmosphere) below the ionization limit.

For Fe I, our model atom was constructed using all known energy levels and the predicted levels with $E_{\text {exc }}$ up to $7.83 \mathrm{eV}$, in total, 2970 levels. Multiplet fine structure was neglected for all terms. The predicted and measured levels with close energies were combined resulting in 233 terms. In addition, six super-levels were made up from the remaining predicted levels. For 11958 radiative transitions occurring in this atom of Fe I, $g f$-values were taken from the Nave et al. (1994) compilation, where available, and Kurucz (2007) calculations. Photoionization cross-sections of the IRON project (Bautista (1997)) have been used for 149 levels and a hydrogenic approximation for the remaining levels. The collisional rates were computed as in Paper I.

For Fe II, we rely on the reference model atom treated in Paper I. In this study, it was reduced and includes now the levels with $E_{\text {exc }}$ up to $10 \mathrm{eV}$. The main uncertainty of the NLTE calculations for Fe I and II is the treatment of the poorly known inelastic collisions with hydrogen atoms. We employ the formula of Steenbock \& Holweger (1984) for allowed transitions and a simple correlation between hydrogen and electron collisional rates, $C_{H}=C_{e} \sqrt{\left(m_{e} / m_{H}\right)} N_{H} / N_{e}$, for forbidden transitions. Calculations were performed with the hydrogen collision enhancement factor $S_{\mathrm{H}}$, which was varied between 0 and 3 .

\section{Results}

The coupled radiative transfer and statistical equilibrium equations are solved with an improved version of the DETAIL program (Butler \& Giddings (1985)) based on the accelerated lambda iteration. All calculations are performed with plane-parallel, homogeneous, LTE, and blanketed model atmospheres computed with the MAFAGS-OS code (Grupp et al. (2009)).

For comparison with observed data, a total of 43 lines of Fe I and 18 lines of Fe II were chosen. For the Sun and HD 84937, the analysis was extended to a larger line list including 271 lines of Fe I and 34 lines of Fe II. The Sun is also used as a reference star for a line-by-line differential analysis of stellar spectra. Solar flux observations were taken from the Kitt Peak Solar Atlas (Kurucz et al. (1984)). The absolute solar iron abundances were determined using $g f$-values from O'Brian et al. (1991) and Meléndez \& Barbuy (2009) for Fe I and Fe II, respectively. We find that virtually all models of line formation, whether LTE or NLTE with $S_{H} \geqslant 0.1$, lead to acceptable solar ionization equilibria within their $1 \sigma$ error bars. To show the maximal NLTE effect on abundance determination, Table 1 presents the average abundances for both ionization stages derived from the NLTE with $S_{H}=0$ (denoted as $\left.\mathrm{NLTE}_{0}\right)$ and LTE calculations. 
Table 1. Stellar parameters and iron abundances obtained for selected stars

\begin{tabular}{|c|c|c|c|c|c|c|c|}
\hline \multirow[t]{2}{*}{ HD } & \multirow[t]{2}{*}{$T_{\text {eff }}$} & \multirow[t]{2}{*}{$\log g$} & \multirow{2}{*}{$\begin{array}{c}V_{\mathrm{mic}} \\
\mathrm{km} \mathrm{s}^{-1}\end{array}$} & \multicolumn{2}{|c|}{$[\mathrm{Fe} / \mathrm{H}]_{\mathrm{I}}$} & \multicolumn{2}{|c|}{$[\mathrm{Fe} / \mathrm{H}]_{\mathrm{II}}$} \\
\hline & & & & $\mathrm{NLTE}_{0}$ & LTE & $\mathrm{NLTE}_{0}$ & LTE \\
\hline Sun & 5777 & 4.44 & 0.9 & $7.63 \pm 0.08$ & $7.49 \pm 0.10$ & $7.44 \pm 0.06$ & $7.45 \pm 0.06$ \\
\hline 10700 & 5377 & 4.53 & 0.8 & $-0.43 \pm 0.04$ & $-0.49 \pm 0.02$ & $-0.53 \pm 0.05$ & $-0.52 \pm 0.05$ \\
\hline 61421 & 6510 & $3.96 \pm 0.02$ & 1.8 & $-0.10 \pm 0.06$ & $-0.14 \pm 0.05$ & $-0.04 \pm 0.03$ & $-0.04 \pm 0.03$ \\
\hline 84937 & 6350 & $4.00 \pm 0.12$ & 1.7 & $-1.94 \pm 0.06$ & $-2.16 \pm 0.07$ & $-2.08 \pm 0.04$ & $-2.11 \pm 0.04$ \\
\hline 102870 & 6060 & $4.11 \pm 0.01$ & 1.4 & $0.04 \pm 0.03$ & $0.04 \pm 0.03$ & $0.13 \pm 0.04$ & $0.12 \pm 0.04$ \\
\hline
\end{tabular}

Four stars with effective temperature and surface gravity measured from the modelindependent methods were chosen to investigate the ionization equilibrium between $\mathrm{Fe}$ I and Fe II for various $S_{H}$ values. They are listed in Table 1 together with the $T_{\text {eff }}$ and $\log$ g values taken from Di Folco et al. (2004) for HD 10700 ( $\tau$ Cet), Allende Prieto et al. (2002) for HD 61421 (Procyon), Korn et al. (2003) for HD 84937, North et al. (2009) for HD 102870 ( $\beta$ Vir). Observational data were obtained with the FOCES spectrograph at the $2.2 \mathrm{~m}$ telescope of the Calar Alto Observatory during a number of observing runs between 1997 and 2005, with a spectral resolution of $R \simeq 60000$ and a signal-to-noise ratio $S / N \geqslant 200$.

The NLTE, $S_{H}=0$ and LTE abundances obtained from the lines of Fe I (denoted as $\left.[\mathrm{Fe} / \mathrm{H}]_{\mathrm{I}}\right)$ and $\mathrm{Fe}$ II $\left([\mathrm{Fe} / \mathrm{H}]_{\mathrm{II}}\right)$ are presented in Table 1 . It is worth noting that, with the updated model atom of Fe I-II, the departures from LTE are substantially smaller compared to those from the previous studies. For example, with $S_{H}=0$, we obtain an average NLTE abundance correction $\Delta_{\mathrm{NLTE}}=\log \varepsilon_{\mathrm{NLTE}}-\log \varepsilon_{\mathrm{LTE}}=0.22$ dex for the Fe I lines in HD 84937, while the corresponding value amounts 0.40 dex in Korn et al. (2003). Figure 1 displays the abundance difference between Fe I and Fe II for various assumptions for the hydrogen collisions. We find that NLTE with pure electronic collisions $\left(S_{H}=0\right)$ is not acceptable for HD 84937 and $\tau$ Cet. This indicates the need for thermalizing processes not involving electrons in the atmosphere of metal-poor stars. For each object, the NLTE effect on abundance determination is small (within the error bars) when hydrogen collisions are included with $S_{H} \geqslant 1$. For Procyon and $\beta$ Vir, the mean Fe abundance from Fe I lines is about 0.1 dex lower compared to that from Fe II

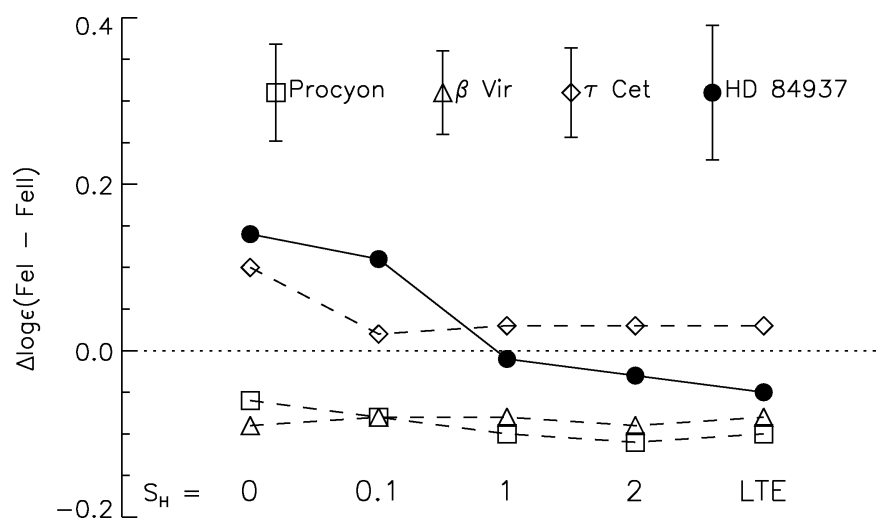

Figure 1. The difference in abundance between Fe I and Fe II in selected stars from calculations with various line formation models. For each star, the error bars is indicated in the upper part of panel. 
lines. The origin of such a discrepancy will be investigated in a forthcoming paper. For the Fe I/Fe II ionization equilibrium in two metal-poor stars, LTE seems to be as good as NLTE with $S_{H} \geqslant 1$.

The NLTE calculations were performed with $S_{H}=1$ for the small grid of model atmospheres with $T_{\text {eff }}=5000$ and $6000 \mathrm{~K},[\mathrm{M} / \mathrm{H}]=-1$ and -3 , and $\log \mathrm{g}$ ranging between 2 and 4 in order to inspect the departures from LTE depending on stellar parameters. Negligible NLTE effects were obtained for Fe II. Fe I is subject to significant NLTE effects for low gravity $(\log g<3$ ) and very metal-poor models. An important consequence is that surface gravities of giants and very metal-poor stars derived by LTE analysis are in error with a magnitude strongly depending on $\log \mathrm{g} /[\mathrm{Fe} / \mathrm{H}]$. For example, LTE leads to a 0.26 dex lower gravity for $T_{\text {eff }}=5000 \mathrm{~K}, \log \mathrm{g}=2$, and $[\mathrm{Fe} / \mathrm{H}]=-3$.

\section{Conclusions}

- Completeness of model atom for Fe I is important for a correct calculation of the $\mathrm{Fe}$ I/Fe II ionization equilibrium in the atmosphere of cool stars.

- Thermalizing processes not involving electron collisions have to be included in the SE calculations for Fe I-II. Collisions with hydrogen atoms could be good candidates for such processes.

- Fe I is affected by significant NLTE effects for giants and very metal-poor stars.

- Only minor departures from LTE are obtained for Fe II.

\section{Acknowledgements}

L.M. acknowledges a partial support from the International Astronomical Union, the Russian Foundation for Basic Research (08-02-92203-GFEN), and the Russian Federal Agency on Science and Innovation (02.740.11.0247) of the participation at the IAU XXVII General Assembly. This study is supported by the Deutsche Forschungsgemeinschaft (GE 490/34.1). A.K. acknowledges support by the Swedish Research Council (VR).

\section{References}

Allende Prieto, C., Asplund, M., Garsia Lopez, R. J., \& Lambert, D. 2002, ApJ, 567, 544

Athay, R. G. \& Lites, B. W. 1972, ApJ, 176, 809

Bautista, M. A. 1997, A\&AS, 122, 167

Butler, K. \& Giddings, J. 1985, Newsletter on the analysis of astronomical spectra, No. 9, University of London

Di Folco, E., Thvenin, F., Kervella, P, et al. 2004, A\&SA, 426, 601

Gehren, T., Butler, K., Mashonkina, L., Reetz, J., \& Shi, J. 2001, A\&A, 366, 981 (Paper I)

Grupp, F., Kurucz, R. L., \& Tan, K. 2009, A\&A, 503, 177

Korn, A., Shi, J. \& Gehren, T. 2003 A\&A, 407, 691

Kurucz, R. 2007, http://kurucz.harvard.edu

Kurucz, R.L., Furenlid, I., Brault, J., \& Testerman, L. 1984, Solar Flux Atlas from 296 to 1300 nm. Nat. Solar Obs., Sunspot, New Mexico

Meléndez, J. \& Barbuy, B. 2009, A\&A, 497, 611

Nave, G., Johansson, S., Learner, R. C. M., Thorne, A. P., \& Brault, J.W. 1994, ApJS, 94, 221

North, J. R., Davis, J., Robertson, J. G., et al. 2009, MNRAS, 393, 245

O'Brian, T. R., Wickliffe, M. E., Lawler, J. E., et al. 1991, J. Opt. Soc. Am. B, 8, 1185

Steenbock, W. \& Holweger, H. 1984, A\& A, 130, 319

Thevenin, F. \& Idiart, T.P. 1999, ApJ, 521, 753 